\title{
Synthesis of novel chiral $\beta$-amino alcohols and diamino alcohols from products of Ugi 3-component reaction
}

\author{
Fu-Lin Chen, Hon-Kai Fu, Chen-Chi Liu, Michael Sung, ${ }^{\dagger}$ and Kuangsen Sung* \\ Department of Chemistry, National Cheng Kung University, Tainan, Taiwan, ROC \\ E-mail: kssung@mail.ncku.edu.tw
}

\begin{abstract}
Novel chiral $\beta$-amino alcohols and diamino alcohols, which are useful in catalytic asymmetric syntheses, were prepared from the products of Ugi 3-component reaction.
\end{abstract}

Keywords. $\beta$-Amino alcohol, diamino alcohol, multi-component reaction

\section{Introduction}

Most catalytic asymmetric syntheses are catalyzed by transition metals in combination with chiral ligands. ${ }^{1}$ Among the popular chiral ligands which have been used for this purpose are $\beta$ amino alcohols. ${ }^{2}$ In addition, chiral diamino alcohols also have been used as ligands in some catalytic asymmetric syntheses. ${ }^{3}$

Some of the chiral $\beta$-amino alcohols are natural products, such as cinchonine, cinchonidine, quinine, quinidine, ephedrine, and norephedrine. ${ }^{4}$ Most of the chiral $\beta$-amino alcohols were prepared from reduction of enantiopure amino acids, ${ }^{5}$ Sharpless' aminohydroxylation of olefins, ${ }^{6}$ Mannich-type reactions for the direct addition of $\alpha$-hydroxy ketones to imines, ${ }^{7}$ regioselective ring opening of chiral epoxides and aziridines, ${ }^{8}$ and nucleophilic addition to $\alpha$-amino carbonyl compounds and $\alpha$-hydroxy imines. ${ }^{9}$

In our previous study, we found that bulky substituents of both aldehydes and $\alpha$-amino acids make Ugi 3-component reaction (U-3CR) highly stereoselective, and several chiral $\alpha, \alpha^{\prime}-$ iminodiacetic acid analogues were prepared by this method easily with high yield. ${ }^{10}$ (Scheme 1 ) By taking advantage of this, we report herein synthesis of novel chiral $\beta$-amino alcohols and diamino alcohols from these chiral $\alpha, \alpha$ '-iminodiacetic acid analogues.

\footnotetext{
${ }^{\dagger}$ : Current address: Riverside Secondary School, 2215 Reeve Street, Port Coquitlam, B.C., Canada V3C 6K8.
} 


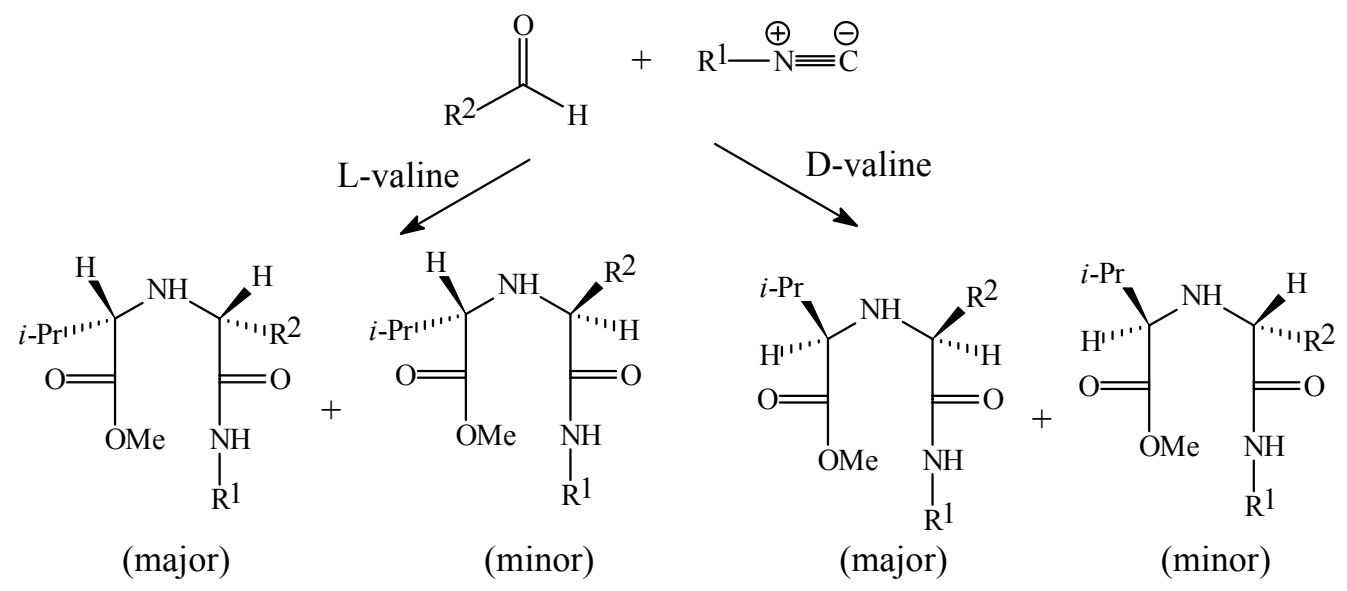

\section{Scheme 1}

\section{Results and Discussion}

As shown in Scheme 2, reduction of $\mathbf{1 a}$ with $\mathrm{LiAlH}_{4}$ in anhydrous ether at room temperature for 20 hours generated 2a with selective reduction of an ester group in a good yield. When the reduction ran at room temperature for 2 days, 1a produced 3a with reduction of both ester and amide groups in a good yield. Under this condition, no racemization products could be detected.

On the other hand, when $\mathrm{LiAlH}_{4}$ reduced $\mathbf{1 b}$ at the same condition, only $\mathbf{2 b}$ could be generated in a moderate yield and no trace of $\mathbf{3 b}$ could be detected. During the reduction, the color changed from yellow to red in 5 minutes, and the reaction was quenched with water. The product $\mathbf{2} \mathbf{b}$ was degraded at a longer reaction time before formation of $\mathbf{3 b}$, which has both ester and amide groups reduced. Therefore, a longer reaction time did not result in the production of $\mathbf{3 b}$, but in the reduction of the yield of $\mathbf{2} \mathbf{b}$.

Methylation of $\mathbf{1 a}$ and $\mathbf{1 b}$ with $\mathrm{MeI}$ in acetonitrile in the presence of $\mathrm{Na}_{2} \mathrm{CO}_{3}$ generated $\mathbf{4 a}$ and $\mathbf{4 b}$, respectively, in good yields. Bulkier alkylating reagents such as benzyl bromide did not react with $\mathbf{1 a}$ or $\mathbf{1 b}$ under the same condition. It is clear that bulky substituents on $\mathbf{1 a}$ and $\mathbf{1 b}$ make them less nucleophilic.

When $4 \mathbf{a}$ was reduced by $\mathrm{LiAlH}_{4}$ in anhydrous ether at room temperature for 2 days, $5 \mathbf{a}$ and $\mathbf{6 a}$ were generated in $39 \%$ and $47 \%$ yields. In contrast, when $\mathrm{LiAlH}_{4}$ reduced $\mathbf{4 b}$ under the same condition, only $\mathbf{5 b}$ could be produced in a moderate yield and no trace of $\mathbf{6 b}$ could be detected. During the reduction, the color changed from yellow to red in 5 minutes and then the reaction was quenched with water. The product $\mathbf{5 b}$ was degraded at a longer reaction time, and as a result, $\mathbf{6 b}$ with reduction of both ester and amide groups could not be obtained at all.

In conclusion, we prepared novel chiral $\beta$-amino alcohols $(\mathbf{2 a}, \mathbf{2 b}, \mathbf{5 a}, \mathbf{5 b})$ and diamino alcohols (3a, 6a) from the products of Ugi 3-component reaction. 


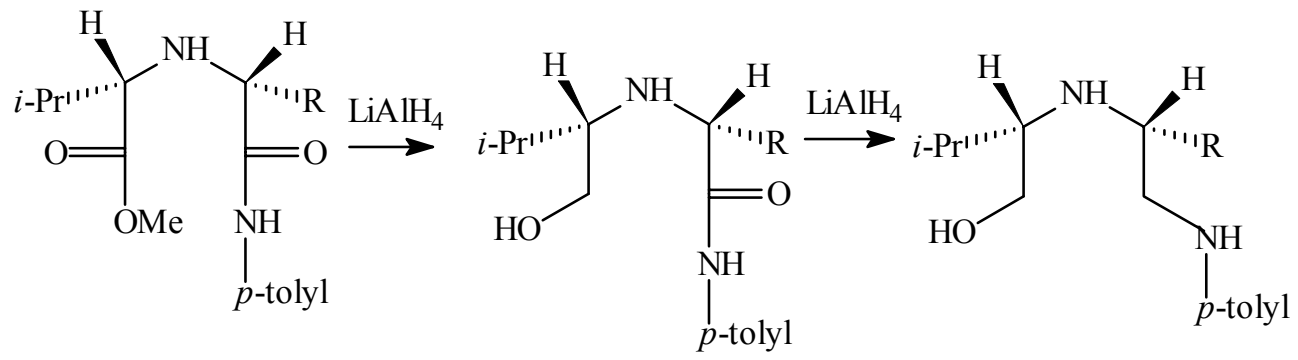

1a,b

$\mathbf{2 a , b}$

3a

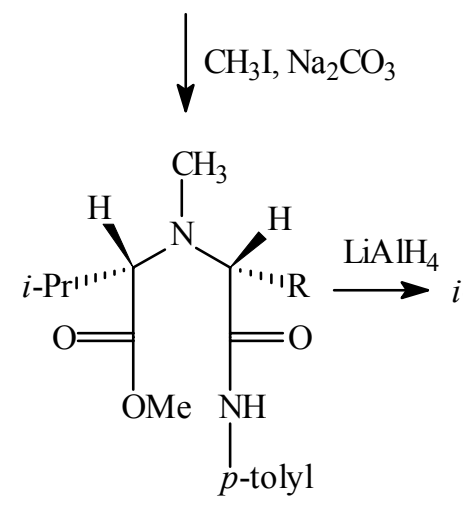

$4 a, b$

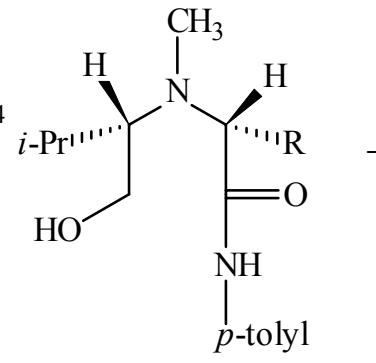

5a,b

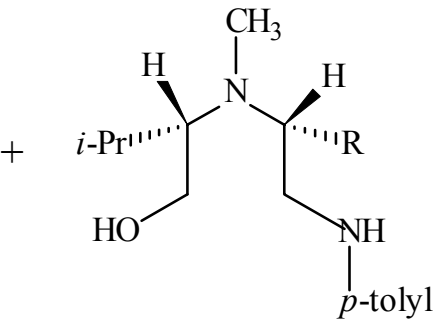

$6 a$

a: $\mathrm{R}=\left(\mathrm{C}_{2} \mathrm{H}_{5}\right)_{2} \mathrm{CH} ; \mathbf{b}: \mathrm{R}=9$-anthracenyl

Scheme 2

\section{Experimental Section}

General Procedures. The starting materials, 1a and 1b, were prepared according the literature method. $^{10}$

Preparation of (2R)-2-(1-Hydroxy-3-methylbutan-(2S)-2-ylamino)-3-ethyl- $N$-ptolylpentanamide (2a). To a solution of 1a $(1.40 \mathrm{~g}, 4 \mathrm{mmol})$ in $25 \mathrm{~mL}$ of dry ethyl ether lithium aluminum hydride (1.52 $\mathrm{g}, 40 \mathrm{mmol})$ was added. The mixture was stirred in a nitrogen atmosphere at room temperature for 20 hours. After the reaction was completed, water was poured into the reaction mixture and the solution was extracted with ethyl ether. The ether layer was dried with anhydrous sodium sulfate and concentrated by a rotary evaporator. The crude product was purified by column chromatography with hexane / ethyl acetate (3:1) as a mobile phase to get $1.04 \mathrm{~g}$ of 2a, colorless oil; yield: $71 \%$; $[\alpha]^{20}{ }_{\mathrm{D}}=+40.8^{\circ}(\mathrm{c}=0.025 \mathrm{~g} / \mathrm{mL}$, ethyl acetate); ${ }^{1} \mathrm{H}$ NMR $\left(\mathrm{CDCl}_{3}\right) \delta 0.87\left(6 \mathrm{H}, \mathrm{m}, \mathrm{CH}_{3}\right), 0.94\left(6 \mathrm{H}, \mathrm{m}, \mathrm{CH}_{3}\right), 1.23 \sim 1.30\left(2 \mathrm{H}, \mathrm{m}, \mathrm{CH}_{2}\right)$, 1.45 1.50 (2H, m, $\left.\mathrm{CH}_{2}\right), 1.86(2 \mathrm{H}, \mathrm{m}, \mathrm{CH}), 2.28\left(3 \mathrm{H}, \mathrm{s}, \mathrm{CH}_{3}\right), 2.40(1 \mathrm{H}, \mathrm{m}, \mathrm{CH}), 3.30(1 \mathrm{H}, \mathrm{d}$, $J=3.1 \mathrm{~Hz}, \mathrm{CH}), 3.42(1 \mathrm{H}, \mathrm{dd}, J=8.0 \& 10.8 \mathrm{~Hz}, \mathrm{CH}), 3.66(1 \mathrm{H}, \mathrm{dd}, J=3.2 \& 10.8 \mathrm{~Hz}, \mathrm{CH}), 7.06$ 
$(2 \mathrm{H}, \mathrm{d}, J=8.3 \mathrm{~Hz}, \mathrm{PhH}), 7.58(2 \mathrm{H}, \mathrm{d}, J=8.3 \mathrm{~Hz}, \mathrm{PhH}) ;{ }^{13} \mathrm{C} \mathrm{NMR}\left(\mathrm{CDCl}_{3}\right) \delta 12.20,12.22,17.10$, $19.61,20.73,22.37,23.44,28.55,45.41,61.59,62.76,64.12,119.44,129.19,133.17,135.58$, 173.64; IR (thin film) $1650(\mathrm{C}=\mathrm{O}) \mathrm{cm}^{-1}$; HRMS (ESI) m/z calcd for $\mathrm{C}_{19} \mathrm{H}_{33} \mathrm{~N}_{2} \mathrm{O}_{2} 321.2542$, found 321.2539 .

Preparation of (2R)-2-(1-Hydroxyl-3-methylbutan-(2S)-2-ylamino)-2-(anthracen- 9-yl)- $N$-ptolylacetamide (2b). To a solution of $\mathbf{1 b}(0.5 \mathrm{~g}, 1.1 \mathrm{mmol})$ in $20 \mathrm{~mL}$ of dry ethyl ether lithium aluminum hydride $(0.21 \mathrm{~g}, 5.5 \mathrm{mmol})$ was added. The mixture was stirred in a nitrogen atmosphere at room temperature for 5 minutes. After the reaction was completed, water was poured into the reaction mixture and the solution was extracted with ethyl ether. The ether layer was dried with anhydrous sodium sulfate and concentrated by a rotary evaporator. The crude product was purified by column chromatography with hexane / ethyl acetate $(2: 1)$ as a mobile phase to get $0.32 \mathrm{~g}$ of $\mathbf{2 b}$, yellow oil; yield: $63 \%$; $[\alpha]^{20}{ }_{\mathrm{D}}=-47.1^{\circ}\left(\mathrm{c}=0.017 \mathrm{~g} / \mathrm{mL}, \mathrm{CHCl}_{3}\right) ;{ }^{1} \mathrm{H}$ $\operatorname{NMR}\left(\mathrm{CDCl}_{3}\right) \delta 0.53\left(3 \mathrm{H}, \mathrm{d}, J=6.8 \mathrm{~Hz}, \mathrm{CH}_{3}\right), 0.61\left(3 \mathrm{H}, \mathrm{d}, J=6.8 \mathrm{~Hz}, \mathrm{CH}_{3}\right), 1.53(1 \mathrm{H}, \mathrm{m}, \mathrm{CH})$, $2.30\left(3 \mathrm{H}, \mathrm{s}, \mathrm{CH}_{3}\right), 2.56(1 \mathrm{H}, \mathrm{m}, \mathrm{CH}), 3.45(1 \mathrm{H}, \mathrm{dd}, J=10.8 \& 7.7 \mathrm{~Hz}, \mathrm{CH}), 3.72(1 \mathrm{H}, \mathrm{dd}, J=10.8$ \& $3.3 \mathrm{~Hz}, \mathrm{CH}), 5.79(1 \mathrm{H}, \mathrm{s}, \mathrm{CH}), 7.08(2 \mathrm{H}, \mathrm{d}, J=8.2 \mathrm{~Hz}, \mathrm{PhH}), 7.47 \sim 7.49$ (6H, m, PhH), 8.04 $(2 \mathrm{H}, \mathrm{d}, J=8.2 \mathrm{~Hz}, \mathrm{PhH}), 8.37(2 \mathrm{H}, \mathrm{d}, J=8.2 \mathrm{~Hz}, \mathrm{PhH}), 8.46(1 \mathrm{H}, \mathrm{s}, \mathrm{PhH}), 9.67(1 \mathrm{H}, \mathrm{s}, \mathrm{NH}) ;{ }^{13} \mathrm{C}$ $\operatorname{NMR}\left(\mathrm{CDCl}_{3}\right) \delta 18.35,19.04,20.80,29.78,60.12,60.19,66.17,119.65,124.98,126.75,128.40$, 129.33, 129.50, 132.32, 133.59, 135.46, 172.77; IR (thin film) $1660(\mathrm{C}=\mathrm{O}) \mathrm{cm}^{-1}$; HRMS (EI) $\mathrm{m} / \mathrm{z}$ calcd for $\mathrm{C}_{28} \mathrm{H}_{31} \mathrm{~N}_{2} \mathrm{O}_{2} 427.2385$, found 427.2382 .

Preparation of (2S)-2-[(2R)-1-(p-toluidino)-3-ethylpentan-2-ylamino]-3-methyl- butan-1-ol (3a). To a solution of $\mathbf{1 a}(1.40 \mathrm{~g}, 4 \mathrm{mmol})$ in $25 \mathrm{~mL}$ of dry ethyl ether lithium aluminum hydride (1.52 g, $40 \mathrm{mmol})$ was added. The mixture was stirred in a nitrogen atmosphere at room temperature for 2 days. After the reaction was completed, water was poured into the reaction mixture and the solution was extracted with ethyl ether. The ether layer was dried with anhydrous sodium sulfate and concentrated by a rotary evaporator. The crude product was purified by column chromatography with hexane / ethyl acetate (3:1) as a mobile phase to get $1.04 \mathrm{~g}$ of 3a, red brown oil; yield: $85 \% ;[\alpha]_{\mathrm{D}}^{20}=-2.3^{\circ}\left(\mathrm{c}=0.043 \mathrm{~g} / \mathrm{mL}\right.$, ethyl acetate); ${ }^{1} \mathrm{H} \mathrm{NMR}$ $\left(\mathrm{CDCl}_{3}\right) \delta 0.91 \sim 0.96\left(12 \mathrm{H}, \mathrm{m}, \mathrm{CH}_{3}\right), 1.19 \sim 1.40\left(4 \mathrm{H}, \mathrm{m}, \mathrm{CH}_{2}\right), 1.41 \sim 1.46(1 \mathrm{H}, \mathrm{m}, \mathrm{CH})$, 1.74 1.80 (1H, m, CH), $2.23\left(3 \mathrm{H}, \mathrm{s}, \mathrm{CH}_{3}\right), 2.42(1 \mathrm{H}, \mathrm{m}, \mathrm{CH}), 2.85(2 \mathrm{H}, \mathrm{m}, \mathrm{CH}), 3.14(1 \mathrm{H}, \mathrm{m}$, $\mathrm{CH}), 3.41(1 \mathrm{H}, \mathrm{dd}, J=4.4 \& 10.8 \mathrm{~Hz}, \mathrm{CH}), 3.55(1 \mathrm{H}, \mathrm{dd}, J=5.6 \& 10.8 \mathrm{~Hz}, \mathrm{CH}), 6.57(2 \mathrm{H}, \mathrm{d}$, $J=8.1 \mathrm{~Hz}, \mathrm{PhH}), 6.99(2 \mathrm{H}, \mathrm{d}, J=8.1 \mathrm{~Hz}, \mathrm{PhH}) ;{ }^{13} \mathrm{C} \mathrm{NMR}\left(\mathrm{CDCl}_{3}\right) \delta 12.47,12.52,18.73,19.54$, 20.37, 22.05, 22.79, 29.48, 44.13, 45.47, 56.38, 61.49, 62.46, 113.51, 126.83, 129.70, 146.23; IR (thin film) 1616, 1521 (aromatic $\mathrm{C}=\mathrm{C}$ ) $\mathrm{cm}^{-1}$; HRMS (ESI) $\mathrm{m} / \mathrm{z}$ calcd for $\mathrm{C}_{19} \mathrm{H}_{35} \mathrm{~N}_{2} \mathrm{O} 307.2749$, found 307.2752.

Preparation of Methyl (2S)-2- $\{N-[(1 R)-1-(p$-tolylcarbamoyl)-2-ethylbutyl $]-N$ methylamino $\}-3-m e t h y l b u t a n o a t e ~(4 a)$ and Methyl (2S)-2-\{(R)- $N$ - $[(p$-tolyl- carbamoyl) (anthracen-9-yl)methyl]- $N$-methyl-amino -3 -methylbutanoate (4b). To a solution of 1 a or $1 \mathbf{b}$ ( $3 \mathrm{mmol})$ and sodium carbonate $(9 \mathrm{mmol})$ in $10 \mathrm{~mL}$ of acetonitrile methyl iodide $(1.27 \mathrm{~g}, 9$ mmol) was added. The mixture was stirred under reflux in a closed system for 24 hours. After the reaction was completed, water was poured into the reaction mixture and the aqueous solution 
was extracted with ethyl acetate. The ethyl acetate solution was dried with anhydrous sodium sulfate and concentrated by a rotary evaporator. The crude product, $\mathbf{4 a}$ or $\mathbf{4 b}$, was purified by column chromatography with hexane / ethyl acetate (83:17) as a mobile phase. 4a: Yield: $88 \%$; yellow solid; m.p.: $80 \sim 83^{\circ} \mathrm{C}$; $[\alpha]^{20}{ }_{\mathrm{D}}=-11.2^{\circ}\left(\mathrm{c}=0.035 \mathrm{~g} / \mathrm{mL}\right.$, ethyl acetate); ${ }^{1} \mathrm{H}$ NMR $\left(\mathrm{CDCl}_{3}\right) \delta$ $0.89\left(9 \mathrm{H}, \mathrm{m}, \mathrm{CH}_{3}\right), 1.07\left(3 \mathrm{H}, \mathrm{d}, \mathrm{J}=\mathrm{Hz}, \mathrm{CH}_{3}\right), 1.25 \sim 1.44\left(4 \mathrm{H}, \mathrm{m}, \mathrm{CH}_{2}\right), 2.00(1 \mathrm{H}, \mathrm{m}, \mathrm{CH}), 2.17$ $(1 \mathrm{H}, \mathrm{m}, \mathrm{CH}), 2.29\left(3 \mathrm{H}, \mathrm{s}, \mathrm{CH}_{3}\right), 2.37\left(3 \mathrm{H}, \mathrm{s}, \mathrm{CH}_{3}\right), 2.94(1 \mathrm{H}, \mathrm{d}, J=10.5 \mathrm{~Hz}, \mathrm{CH}), 3.01(1 \mathrm{H}, \mathrm{d}$, $J=6.8 \mathrm{~Hz}, \mathrm{CH}), 3.54\left(3 \mathrm{H}, \mathrm{s}, \mathrm{CH}_{3}\right), 7.10(2 \mathrm{H}, \mathrm{d}, J=8.1 \mathrm{~Hz}, \mathrm{PhH}), 7.41(2 \mathrm{H}, \mathrm{d}, J=8.1 \mathrm{~Hz}, \mathrm{PhH})$, $7.95(1 \mathrm{H}, \mathrm{s}, \mathrm{NH}) ;{ }^{13} \mathrm{C} \mathrm{NMR}\left(\mathrm{CDCl}_{3}\right) \delta 10.79,11.73,19.46,19.98,20.78,21.17,21.88,27.60$, $32.52,39.82,51.06,70.77,71.14,119.40,129.42,133.53,135.10,169.61,172.89$; IR (thin film) 1734, $1653(\mathrm{C}=\mathrm{O}) \mathrm{cm}^{-1}$; HRMS (ESI) m/z calcd for $\mathrm{C}_{21} \mathrm{H}_{35} \mathrm{~N}_{2} \mathrm{O}_{3} 363.2648$, found 363.2650. 4b: Yield: 93\%; yellow oil; $[\alpha]^{20}{ }_{\mathrm{D}}=-79.9^{\circ}\left(\mathrm{c}=0.011 \mathrm{~g} / \mathrm{mL}\right.$, ethyl acetate); ${ }^{1} \mathrm{H}$ NMR $\left(\mathrm{CDCl}_{3}\right) \delta 0.58$ $\left(3 \mathrm{H}, \mathrm{d}, J=6.5 \mathrm{~Hz}, \mathrm{CH}_{3}\right), 1.19\left(3 \mathrm{H}, \mathrm{d}, J=6.5 \mathrm{~Hz}, \mathrm{CH}_{3}\right), 2.11(1 \mathrm{H}, \mathrm{m}, \mathrm{CH}), 2.28\left(3 \mathrm{H}, \mathrm{s}, \mathrm{CH}_{3}\right), 2.70$ $\left(3 \mathrm{H}, \mathrm{s}, \mathrm{CH}_{3}\right), 2.93(1 \mathrm{H}, \mathrm{d}, J=9.0 \mathrm{~Hz}, \mathrm{CH}), 3.01\left(3 \mathrm{H}, \mathrm{s}, \mathrm{CH}_{3}\right), 5.78(1 \mathrm{H}, \mathrm{s}, \mathrm{CH}), 7.08(2 \mathrm{H}, \mathrm{d}, J=4.1$ $\mathrm{Hz}, \mathrm{PhH}), 7.35 \sim 7.55$ (6H, m, PhH), 8.01 (2H, d, J=4.2 Hz, PhH), 8.50 (3H, m, PhH), $8.81(1 \mathrm{H}, \mathrm{s}$, $\mathrm{NH}) ;{ }^{13} \mathrm{C} \mathrm{NMR}\left(\mathrm{CDCl}_{3}\right) \delta 19.60,20.03,20.81,28.54,36.91,49.90,67.14,68.28,119.53,124.86$, $126.71,127.42,129.46,129.50,129.73,131.61,133.85,135.07,170.00,173.38$; IR (thin film) 1728, $1694(\mathrm{C}=\mathrm{O}) \mathrm{cm}^{-1}$; HRMS (EI) $\mathrm{m} / \mathrm{z}$ calcd for $\mathrm{C}_{30} \mathrm{H}_{33} \mathrm{~N}_{2} \mathrm{O}_{3} 469.2491$, found 469.2487 .

Preparation of (2R)-2-[N-(1-Hydroxy-3-methylbutan-(2S)-2-yl)- $N$-methylamino]- 3-ethyl$N$-p-tolylpentanamide (5a) and (2S)-2- $\{N-[(2 R)-1-(p$-toluidino)-3-ethyl- pentan-2-yl]- $N$ methylamino -3-methylbutan-1-ol (6a). To a solution of $4 \mathbf{a}(2.17 \mathrm{~g}, 6 \mathrm{mmol})$ in $30 \mathrm{~mL}$ of dry ethyl ether lithium aluminum hydride $(2.28 \mathrm{~g}, 60 \mathrm{mmol})$ was added. The mixture was stirred in a nitrogen atmosphere at room temperature for 2 days. After the reaction was completed, water was poured into the reaction mixture and the solution was extracted with ethyl ether. The ether layer was dried with anhydrous sodium sulfate and concentrated by a rotary evaporator. The crude product was purified by column chromatography with hexane / ethyl acetate (83:17) as a mobile phase to get $0.781 \mathrm{~g}$ of $5 \mathbf{a}$ and $0.902 \mathrm{~g}$ of $\mathbf{6 a}$. 5a: Yield: $39 \%$; colorless oil; $[\alpha]^{20}{ }_{\mathrm{D}}=+2.9^{\circ}$ (c=0.034 g/mL, ethyl acetate); ${ }^{1} \mathrm{H}$ NMR $\left(\mathrm{CDCl}_{3}\right) \delta 0.92\left(6 \mathrm{H}, \mathrm{m}, \mathrm{CH}_{3}\right), 1.06\left(6 \mathrm{H}, \mathrm{m}, \mathrm{CH}_{3}\right)$, 1.53 1.61 (4H, m, CH$), 1.75(1 \mathrm{H}, \mathrm{m}, \mathrm{CH}), 1.86(1 \mathrm{H}, \mathrm{m}, \mathrm{CH}), 2.29\left(3 \mathrm{H}, \mathrm{s}, \mathrm{CH}_{3}\right), 2.37(3 \mathrm{H}, \mathrm{s}$, $\left.\mathrm{CH}_{3}\right), 2.49(1 \mathrm{H}, \mathrm{m}, \mathrm{CH}), 3.41(1 \mathrm{H}, \mathrm{d}, J=4.7 \mathrm{~Hz}, \mathrm{CH}), 3.74(2 \mathrm{H}, \mathrm{d}, J=6.0 \mathrm{~Hz}, \mathrm{CH}), 7.10(2 \mathrm{H}, \mathrm{d}$, $J=8.1 \mathrm{~Hz}, \mathrm{PhH}), 7.44(2 \mathrm{H}, \mathrm{d}, J=8.1 \mathrm{~Hz}, \mathrm{PhH}), 8.74(1 \mathrm{H}, \mathrm{s}, \mathrm{NH}) ;{ }^{13} \mathrm{C} \mathrm{NMR}\left(\mathrm{CDCl}_{3}\right) \delta 12.12$, 12.16, 20.40, 20.81, 21.86, 22.51, 22.76, 28.19, 30.89, 32.09, 41.73, 60.97, 67.06, 70.22, 119.51, 129.39, 133.38, 135.44, 171.42; IR (thin film) $1653(\mathrm{C}=\mathrm{O}) \mathrm{cm}^{-1}$; HRMS (ESI) $\mathrm{m} / \mathrm{z}$ calcd for $\mathrm{C}_{20} \mathrm{H}_{35} \mathrm{~N}_{2} \mathrm{O}_{2}$ 335.2698, found 335.2696. 6a: Yield: 47\%; light red oil; $[\alpha]^{20}{ }_{\mathrm{D}}=-6.5^{\circ}(\mathrm{c}=0.015$ $\mathrm{g} / \mathrm{mL}$, ethyl acetate); ${ }^{1} \mathrm{H}$ NMR $\left(\mathrm{CDCl}_{3}\right) \delta 0.87 \sim 0.98\left(12 \mathrm{H}, \mathrm{m}, \mathrm{CH}_{3}\right), 1.35 \sim 1.48\left(4 \mathrm{H}, \mathrm{m}, \mathrm{CH}_{2}\right)$, $1.61(1 \mathrm{H}, \mathrm{m}, \mathrm{CH}), 1.82(1 \mathrm{H}, \mathrm{m}, \mathrm{CH}), 2.23\left(3 \mathrm{H}, \mathrm{s}, \mathrm{CH}_{3}\right), 2.39\left(3 \mathrm{H}, \mathrm{s}, \mathrm{CH}_{3}\right), 2.55(1 \mathrm{H}, \mathrm{m}, \mathrm{CH})$, $2.93(1 \mathrm{H}, \mathrm{m}, \mathrm{CH}), 3.07(1 \mathrm{H}, \mathrm{dd}, J=3.2 \& 12.7 \mathrm{~Hz}, \mathrm{CH}), 3.20(1 \mathrm{H}, \mathrm{dd}, J=9.4 \& 12.7 \mathrm{~Hz}, \mathrm{CH})$, $6.57(2 \mathrm{H}, \mathrm{d}, J=8.0 \mathrm{~Hz}, \mathrm{PhH}), 6.99(2 \mathrm{H}, \mathrm{d}, J=8.0 \mathrm{~Hz}, \mathrm{PhH}) ;{ }^{13} \mathrm{C} \mathrm{NMR}\left(\mathrm{CDCl}_{3}\right) \delta 12.01,12.47$, $19.69,20.39,21.87,22.40,23.68,29.19,29.48,43.58,44.90,60.45,65.08,70.44,113.86$, 127.33, 129.72, 146.10; IR (thin film) $3313(\mathrm{NH}) \mathrm{cm}^{-1}$; HRMS (ESI) m/z calcd for $\mathrm{C}_{20} \mathrm{H}_{37} \mathrm{~N}_{2} \mathrm{O}$ 321.2906, found 321.2904 . 
Preparation of (2R)-2-[N-(1-Hydroxy-3-methylbutan-(2S)-2-yl)- $N$-methylamino]- 2(anthracen-9-yl)- $\boldsymbol{N}$-p-tolylacetamide (5b). To a solution of $\mathbf{4 b}(0.2 \mathrm{~g}, 0.42 \mathrm{mmol})$ in $10 \mathrm{~mL}$ of dry ethyl ether lithium aluminum hydride $(0.1 \mathrm{~g}, 2.4 \mathrm{mmol})$ was added. The mixture was stirred in a nitrogen atmosphere at room temperature for 5 minutes. After the reaction was completed, water was poured into the reaction mixture and the solution was extracted with ethyl ether. The ether layer was dried with anhydrous sodium sulfate and concentrated by a rotary evaporator. The crude product was purified by column chromatography with chloroform / ethyl acetate (94:6) as a mobile phase to get $0.12 \mathrm{~g}$ of $\mathbf{5 b}$, colorless oil; yield: $62 \% ;[\alpha]^{20}{ }_{\mathrm{D}}=-107.7^{\circ}(\mathrm{c}=0.007$ $\mathrm{g} / \mathrm{mL}$, chloroform); ${ }^{1} \mathrm{H} \mathrm{NMR}\left(\mathrm{CDCl}_{3}\right) \delta 0.93\left(3 \mathrm{H}, \mathrm{d}, J=6.8 \mathrm{~Hz}, \mathrm{CH}_{3}\right), 1.23(3 \mathrm{H}, \mathrm{d}, J=6.8 \mathrm{~Hz}$, $\left.\mathrm{CH}_{3}\right), 2.08(1 \mathrm{H}, \mathrm{m}, \mathrm{CH}), 2.21\left(3 \mathrm{H}, \mathrm{s}, \mathrm{CH}_{3}\right), 2.24\left(3 \mathrm{H}, \mathrm{s}, \mathrm{CH}_{3}\right), 3.03(1 \mathrm{H}, \mathrm{m}, \mathrm{CH}), 3.73(2 \mathrm{H}, \mathrm{m}$, $\left.\mathrm{CH}_{2}\right), 6.14(1 \mathrm{H}, \mathrm{s}, \mathrm{CH}), 7.01(2 \mathrm{H}, \mathrm{d}, J=4.2 \mathrm{~Hz}, \mathrm{PhH}), 7.26(2 \mathrm{H}, \mathrm{s}, \mathrm{PhH}), 7.47 \sim 7.55$ (5H, m, $\mathrm{PhH}), 8.02$ (2H, d, J=4.2 Hz, PhH), $8.27(1 \mathrm{H}, \mathrm{s}, \mathrm{PhH}), 8.49(1 \mathrm{H}, \mathrm{s}, \mathrm{PhH}), 8.61(1 \mathrm{H}, \mathrm{s}, \mathrm{NH}) ;{ }^{13} \mathrm{C}$ NMR $\left(\mathrm{CDCl}_{3}\right) \delta 20.07,20.78,23.25,26.95,27.91,28.00,31.82,60.48,66.16,68.82,119.89$, $125.09,127.09,128.42,129.28,129.47,131.67,133.82,143.52,171.94,175.44$; IR (thin film) $1653(\mathrm{C}=\mathrm{O}) \mathrm{cm}^{-1}$; HRMS (EI) $\mathrm{m} / \mathrm{z}$ calcd for $\mathrm{C}_{29} \mathrm{H}_{33} \mathrm{~N}_{2} \mathrm{O}_{2} 441.2542$, found 441.2545.

\section{Acknowledgments}

Financial support by the National Science Council of Taiwan (NSC 94-2113-M-006-009) is gratefully acknowledged.

\section{References}

1. (a) Noyori, R. Adv. Synth. Catal. 2003, 345, 15. (b) Corey, E. J.; Helal, C. J. Angew. Chem. Int. Ed. 1998, 37, 1986. (c) McGarrigle, E. M.; Gilheany, D. G. Chem. Rev. 2005, 105, 1563. (d) Walsh, P. J. Acc. Chem. Res. 2003, 36, 739.

2. (a) Burguete, M. I.; Garcia-Verdugo, E.; Vicent, M. J.; Luis, S. V.; Pennemann, H.; von Keyserling, N. G.; Martens, J. Org. Lett. 2002, 4, 3947. (b) Puigjaner, C.; Vidal-Ferran, A.; Moyano, A.; Pericas, M. A.; Riera, A. J. Org. Chem. 1999, 64, 7902. (c) Sola, L.; Reddy, K. S.; Vidal-Ferran, A.; Moyano, A.; Pericas, M. A.; Riera, A.; Alvarez-Larena, A.; Piniella, J. F. J. Org. Chem. 1998, 63, 7078. (d) Yamakawa, M.; Noyori, R. J. Am. Chem. Soc. 1995, 117, 6327. (e) Soai, K.; Niwa, S. Chem. Rev. 1992, 92, 833. (f) Corey, E. J.; Bakshi, R. K.; Shibata, S. J. Am. Chem. Soc. 1987, 109, 5551.

3. (a) Corey, E. J.; Naef, R.; Hannon, F. J. J. Am. Chem. Soc. 1986, 108, 7114. (b) Corey, E. J.; Hannon, F. J. Tetrahedron Lett. 1987, 28, 5233. (c) Tanaka, K.; Ushio, H.; Suzuki, H. J. Chem. Soc., Chem. Commun. 1989, 1700. (d) Corey, E. J.; Yuen, P.-W.; Hannon, F. J.; Wierda, D. A. J. Org. Chem. 1990, 55, 784.

4. (a) Studer, M.; Blaser, H.-U.; Exner, C. Adv. Synth. Catal. 2003, 345, 45. (b) Smaardijk, A. 
A.; Wynberg, H. J. Org. Chem. 1987, 52, 135. (c) Wynberg, H. Asymmetric Catalysis by Cinchona Alkaloids. In Topics in Stereochemistry; Eliel, E. L., Wilen, S., Eds.; WileyInterscience: New York, 1986; Vol. 16. (d) Muchow, G.; Vannoorenberghe, Y.; Buono, G. Tetrahedron Lett. 1987, 28, 6163. (e) Chaloner, P. A.; Langadianou, E. Tetrahedron Lett. 1987, 28, 3013. (f) Corey, E. J.; Hannon, F. J. Tetrahedron Lett. 1987, 28, 5237. (g) Soai, K.; Yokoyama, S.; Ebihara, K.; Hayasaka, T. J. Chem. Soc., Chem. Commun. 1987, 1690. (h) Soai, K.; Yokoyama, S.; Hayasaka, T. J. Org. Chem. 1991, 56, 4264.

5. (a) Burguete, M. I.; Garcia-Verdugo, E.; Vincent, M. J.; Luis, S. V.; Pennemann, H.; von Keyserling, N. G.; Martens, J. Org. Lett. 2002, 4, 3947. (b) Soai, K.; Ookawa, A.; Kaba, T.; Ogawa, K. J. Am. Chem. Soc. 1987, 109, 7111. (c) Soai, K.; Ookawa, A. J. Chem. Soc., Chem. Commun. 1986, 412. (d) Ookawa, A.; Soai, K. J. Chem. Soc., Perkin Trans. 1 1987, 1465.

6. (a) O'Brien, P. Angew. Chem. Int. Ed. 1999, 38, 326. (b) Li, G.; Chang, H.-T.; Sharpless, K. B. Angew. Chem. Int. Ed. 1996, 35, 451.

7. (a) Trost, B. M.; Terrell, L. R. J. Am. Chem. Soc. 2003, 125, 338. (b) Cordova, A.; Notz, W.; Zhong, G.; Betancort, J. M., Barbas, C. F., III. J. Am. Chem. Soc. 2002, 124, 1842. (c) List, B. J. Am. Chem. Soc. 2000, 122, 9336.

8. (a) Bartoli, G.; Bosco, M.; Carlone, A.; Locatelli, M.; Massaccesi, M.; Melchiorre, P.; Sambri, L. Org. Lett. 2004, 6, 2173. (b) Chini, M.; Crotti, P.; Flippin, L. A.; Gardelli, C.; Giovani, E.; Macchia, F.; Pineschi, M. J. Org. Chem. 1993, 58, 1221.

9. Bergmeier, S. C. Tetrahedron 2000, 56, 2561.

10. Sung, K.; Chen, F.-L.; Chung, M.-J. Molecular Diversity 2003, 6, 213. 